Discussion This investigation suggests a positive effect of the simultaneous QI development on guideline content concerning specificity of recommendations, clarity of aims to improve quality of care and identification of clinical questions to be addressed in future systematic reviews and/or guidelines.

Implications for Guideline Developers A simultaneous process to develop guidelines and QI is favourable not only to facilitate the assessment of guideline implementation and impact but also to improve guideline content and implementability.

\section{FEASIBILITY AND EFFICIENCY OF STRATEGIES FOR UPDATING CLINICAL PRACTICE GUIDELINES}

${ }^{1} \mathrm{~L}$ Martínez García, 'A Sanabria, ${ }^{2}$ Araya, ${ }^{1} R$ Vernooij, ${ }^{1}$ I Solàà, ${ }^{4}$ J Lawson, ${ }^{4} T$ Navarro, ${ }^{3}$ B Haynes, ${ }^{4} \mathrm{~J}$ Gracia San Román, , ${ }^{5}$ A Kotzeva, ${ }^{6}$ T Marti, ${ }^{1} \mathrm{P}$ Alonso-Coello. ${ }^{1}$ Iberoamerican Cochrane Centre - IIB Sant Pau, Barcelona, Spain; '²Evidence Based Dentistry Unit, Faculty of Dentistry, Universidad de Chile, Chile; ${ }^{3}$ McMaster University, Hamilton, Canada; ${ }^{4}$ Director of the Scientific Committee of Guiasalud, Spain; ${ }^{5}$ Catalan Agency for Health Information, Assessment and Quality (CAHIAQ), Barcelona, Spain; ${ }^{6}$ Axencia de Avaliación de Tecnoloxías Sanitarias de Galicia (avalia-t) Santiago de Compostela, Spain

\section{0:1136/bmjqs-2013-002293.54}

Background Exhaustive search strategies (ESS) for updating clinical practice guidelines (CPGs) recommendations are laborious and expensive. Highly sensitive and specific alternative search strategies are necessary to improve the efficiency in recommendations updating.

Objectives To compare alternative search strategies against ESS Methods We ran three different search strategies in a convenience sample of four CPGs from the CPGs National Programme in Spain: 1) Original ESS (gold standard); 2) Search strategy in the McMaster Premium LiteratUre Service (PLUS) database; and 3) Restrictive strategy with the least number of MeSH terms and text words from the original ESS. We retrieved the key references (which triggered an update) from the original ESS and evaluated their presence in the PLUS and restrictive strategies results. We calculated the sensitivity, specificity, precision, and accuracy for the PLUS and restrictive strategies compared to the ESS.

Results The overall number of references in the PLUS strategy was lower than in the ESS $(39,133$ versus 2,635). The PLUS strategy retrieved a range of $1.12 \%$ to $12.1 \%$ of the total number of references retrieved by the ESS per guideline.

Discussion Our project assessed two novel restrictive search strategies for the updating of CPGs, which could reduce the workload while displaying similar results. Full final findings of this project will be presented at the GIN meeting.

Implications for Guideline Developers/Users Our project has important implications for updating CPGs, informing on the feasibility and efficiency of two novel search strategies.

\section{MAXIMISING EFFICIENCY IN UPDATING GUIDELINES THROUGH PRIORITISATION OF CLINICAL QUESTIONS}

${ }^{1} \mathrm{Q}$ Amos, ${ }^{2} \mathrm{~W}$ Chan, ${ }^{1} \mathrm{G}$ Tom. ${ }^{1}$ Kaiser Permanente, Care Management Institute, Oakland, USA, Kaiser Permanente; ${ }^{2}$ Kaiser Permanente, NW Permanente Group, Oregon, USA

\section{0:1136/bmjgs-2013-002293.55}

Background To maximise efficiency in updating clinical guidelines it is important to understand which of its component clinical questions impact patient care most. Evaluating, editing, and prioritising of each clinical question is required to identify those that warrant updating.
Objectives To describe the methods used by a US health care delivery organisation to prioritise questions within an integrated cardiovascular guideline to determine those that were most important for updating.

Methods 127 clinical questions within an integrated cardiovascular guideline were ranked (using a Likert scale of 1-9) by importance for literature monitoring by clinical experts in each disease domain of the guideline. Examples of factors that influenced rankings included existence of high quality systematic reviews, knowledge that current evidence was relatively unchanged, and the notion that the question was no longer clinically relevant. Questions ranked 7-9 in importance for literature monitoring were considered most important for updating. Conversely, questions with low rankings were considered for retirement.

Results Of 127 questions ranked, 16 were identified as important for literature updating; 12 were retired. We were able to address the most important questions and avoid updating delays of 6-18 months.

Discussion Having these questions prioritised at the outset of updating allowed the healthcare organisation to ensure that the most important clinical questions were being addressed thus making the most efficient use of resources.

Implications for Guideline Developers/Users Evaluating, editing, and prioritising clinical questions improves efficiency when updating guidelines.

\section{THE EFFICIENCY-VALIDITY METHODOLOGICAL CONTINUUM (EVMC) FOR SUSTAINABLE GUIDELINE (CPG) DEVELOPMENT: A NEGOTIATING TOOL FOR CREDIBLE COMPROMISES IN QUALITY FOR AFFORDABLE TRUSTWORTHY GUIDELINES}

${ }^{1} \mathrm{G}$ Browman, ${ }^{2} \mathrm{M}$ Somerfield, ${ }^{3} \mathrm{G}$ Lyman. ${ }^{1}$ British Commbia Cancer Agency, Vancouver Island Center, Victoria, BC, Canada; ${ }^{2}$ American Society of Clinical Oncology, Alexandria, VA, United States of America; ${ }^{3}$ Duke University, Durham, NC, United States of America

\section{0:1136/bmjgs-2013-002293.56}

Background Research methodologists and guideline sponsors are on a collision course as growing demands for scientific rigour raise costs and lengthen delays in CPG production.

Objectives To design a tool for CPG developers and sponsors to negotiate methodological compromises while preserving CPG trustworthiness. There are already variations in CPG quality that we tolerate. Flawless systematic reviews and guidelines are unrealistic. Methodological compromises are inevitable and imposed by practical constraints. Negotiating and reporting methodological compromises can fill a transparency gap where methodological choices are made in the development of a CPG.

Methods Three individuals with guideline development experience collaborated to design a tool that aligns stakeholders' interests while preserving 'trustworthiness' and enhancing transparency.

Results The Efficiency-Validity Methodological Continuum (EVMC) is anchored at the extremes by "practical" at the "efficiency" pole and "best achievable" at the "validity" pole, highlighting the tradeoffs. The continuum between these is represented as a solid line. A 'zone of preference' closer to the 'validity' and a 'zone of acceptability' closer to efficiency are negotiating zones. Beyond the anchors, represented as broken lines, are "expedience" at the efficiency end and "ideal" at the validity end. Guideline development should operate within the solid segment of the continuum. The broken segment towards 